\title{
ONE-DIMENSIONAL BUBBLY CAVITATING FLOWS THROUGH A CONVERGING-DIVERGING NOZZLE
}

\author{
Yi-Chun Wang \\ Department of Mechanical Engineering, \\ National Cheng Kung University, Tainan 701, Taiwan \\ (06)275-7575 ext. 62125, 06-2352973 FAX, wangyc@mail.ncku.edu.tw \\ Christopher E. Brennen \\ California Institute of Technology, Pasadena, California 91125, USA \\ (818)395-4117, 818-5862719 FAX, brennen@ccosun.caltech.edu
}

\begin{abstract}
A non-barotropic continuum bubbly mixture model is used to study the one-dimensional cavitating flow through a converging-diverging nozzle. The nonlinear dynamics of the cavitation bubbles are modeled by the Rayleigh-Plesset equation. Analytical results show that the bubble/bubble interaction through the hydrodynamics of the surrounding liquid has important effects on this confined flow field. One clear interaction effect is the Bernoulli effect caused by the growing and collapsing bubbles in the nozzle. It is found that the characteristics of the flow change dramatically even when the upstream void fraction is very small. Two different flow regimes are found from the steady state solutions and are termed: quasi-steady and quasi-unsteady. The former is characterized by large spatial fluctuations downstream of the throat which are induced by the pulsations of the cavitation bubbles. The quasi-unsteady solutions correspond to flashing flow. Bifurcation occurs as the flow transitions from one regime to the other. An analytical expression for the critical bubble size at the bifurcation is obtained. Physical reasons for this quasi-static instability are also discussed.
\end{abstract}

\section{NOMENCLATURE}

A Dimensionless cross-sectional area of nozzle, $A^{*} / A_{s}^{*}$

$A^{*} \quad$ Cross-sectional area of nozzle

$A_{s}^{*} \quad$ Upstream cross-sectional area of nozzle
$C_{P} \quad$ Pressure coefficient, $\left(p^{*}-p_{s}^{*}\right) / \frac{1}{2} \rho_{L}^{*} u_{s}^{*^{2}}$

$C_{P c} \quad$ Critical pressure coefficient at which flashing occurs

$C_{P M I N}$ Minimum pressure coefficient at throat for pure liquid nozzle flow

$L \quad$ Dimensionless length of the nozzle

$L^{*} \quad$ Length of the nozzle

$R \quad$ Dimensionless bubble radius, $R^{*} / R_{s}^{*}$

$R_{c} \quad$ Dimensionless critical bubble radius at which flashing occurs

$R_{s}^{*} \quad$ Upstream bubble radius

Re Reynolds number, $\rho_{L}^{*} u_{s}^{*} R_{s}^{*} / \mu_{L}^{*}$

$S^{*} \quad$ Surface tension of the liquid

We Weber number, $\rho_{L}^{*} u_{s}^{*^{2}} R_{s}^{*} / S^{*}$

$k \quad$ Polytropic index for the gas inside the bubbles

$p^{*} \quad$ Fluid pressure

$p_{s}^{*} \quad$ Upstream pressure

$p_{V}^{*} \quad$ Vapor pressure

$t \quad$ Dimensionless time, $t^{*} u_{s}^{*} / R_{s}^{*}$

$t^{*} \quad$ Time

$u \quad$ Dimensionless fluid velocity, $u^{*} / u_{s}^{*}$

$u^{*} \quad$ Fluid velocity

$u_{s}^{*} \quad$ Upstream velocity

$x \quad$ Dimensionless Eulerian coordinate, $x^{*} / R_{s}^{*}$

$x^{*} \quad$ Eulerian Coordinate

$\alpha \quad$ Void fraction of the bubbly mixture

$\alpha_{b} \quad$ Upstream void fraction at which flashing occurs

$\alpha_{s} \quad$ Upstream void fraction 
$\eta \quad$ Dimensionless bubble population per unit liquid volume, $\eta^{*} R_{s}^{*^{3}}$

$\eta^{*} \quad$ Bubble population per unit liquid volume

$\gamma \quad$ Ratio of specific heats of the gas inside the bubbles

$\mu_{L}^{*} \quad$ Effective dynamic viscosity of the liquid

$\rho \quad$ Dimensionless fluid density

$\rho_{L}^{*} \quad$ Density of the liquid

$\rho_{s}^{*} \quad$ Upstream mixture density

$\sigma \quad$ Cavitation number, $\left(p_{s}^{*}-p_{V}^{*}\right) / \frac{1}{2} \rho_{L}^{*} u_{s}^{*^{2}}$

\section{INTRODUCTION}

One-dimensional bubbly liquid flows in ducts and nozzles represent one of the simplest confined gas-liquid flows. This is an important problem by itself in many engineering applications, but has not, previously, been studied in the context of cavitation bubble/bubble interactions. The nozzle flow is also a useful model of any cavitating flow in which a low pressure region causes the flow to accelerate, for example, the cavitating flow on the suction surface of a hydrofoil. Therefore, study of the one-dimensional accelerating flow with bubble cavitation effects may have value in building up fully nonlinear solutions for practical three-dimensional flows.

In some flows it is possible to establish a barotropic relation, $p=f(\rho)$, which assumes that the mixture pressure is the function of mixture density only. This implies that all effects caused by bubble content are disregarded except for the compressibility and that the mixture can be regarded effectively as a single-phase compressible fluid. Tangren et. al. (1949) first addressed the barotropic nozzle flow of a twophase mixture. A summary of this subject can be found, for example, in Brennen (1995). In many practical flows, however, the barotropic criterion is not met. In the present context, the dynamic effects of the flow acceleration cause the bubbles to cavitate and then the flow deceleration makes them collapse. Under these circumstances, the mixture is not barotropic and, as we shall see, the growth and collapse of cavitating bubbles can dramatically change or destabilize the flow.

The flow model used here is a nonlinear continuum bubbly mixture model coupled with the Rayleigh-Plesset equation for the bubble dynamics. This model was first proposed by van Wijngaarden $(1968,1972)$ and has been used for studying steady and transient shock wave propagation in bubbly liquids without the acceleration of the mean flow (see, for example, Noordzij and Wijngaarden 1974; Kameda and Matsumoto 1995). Only a few papers have addressed problems with flowing bubbly liquids. Ishii et. al. (1993) proposed a bubbly flow model and used it to study steady

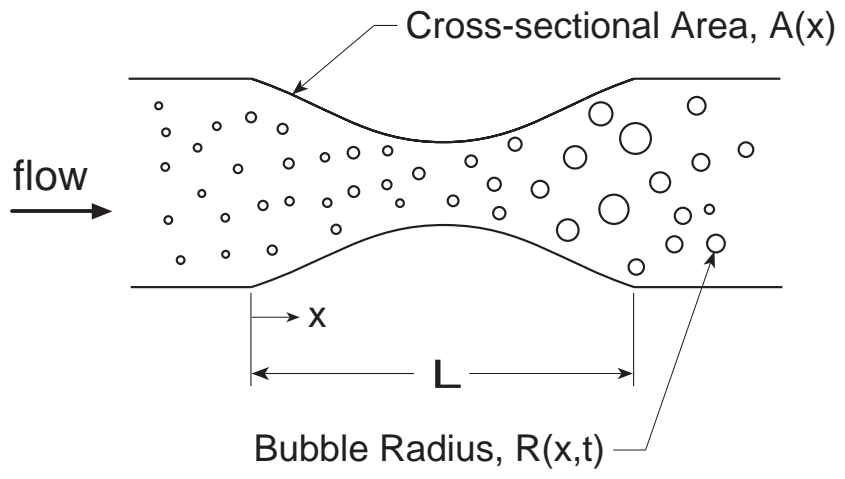

Figure 1: Notation for bubbly liquid flow in a convergingdiverging nozzle.

flows through a converging-diverging nozzle. However, by assuming that the gas pressure inside the bubbles is equal to the ambient fluid pressure, they neglected the bubble radial dynamics (as represented by the Rayleigh-Plesset equation) which are dominant mechanisms in a cavitating flow. Morioka and Matsui (1980) and Morioka and Toma (1984) investigated the acoustic dispersion relation for a flowing bubbly liquid using van Wijngaarden's model and Toma and Morioka (1986) examined characteristics of different acoustic modes in flowing bubbly liquid using the same model. Toma et. al. (1988) conducted experiments with bubbly liquid flows in a converging-diverging nozzle and recorded the temporal fluctuation characteristics of this kind of flow. However, fully nonlinear solutions of the accelerating bubbly flows with bubble cavitation effects have not, previously, been obtained. The purpose of the present work is to examine what effects bubble dynamics can have on the flow structure.

\section{BASIC EQUATIONS}

Referring to figure 1, consider a one-dimensional converging-diverging nozzle with length $L$ and crosssectional area $A(x)$. The flow direction is in positive $x$ direction and the inlet of the nozzle is located at $x=0$.

The variables in all the figures and equations are nondimensionalized using the upstream conditions (denoted by subscript $s$ ) and the liquid density. All quantities with superscript * represent dimensional values. For example, $\eta=\eta^{*} R_{s}^{*^{3}}$ is the non-dimensional bubble population per unit liquid volume, where $R_{s}^{*}$ is upstream bubble radius.

The continuity and momentum equations of the bubbly flow (references d'Agostino and Brennen 1983, 1989; Wang 1996) have the forms

$$
\frac{\partial}{\partial t}[(1-\alpha) A]+\frac{\partial}{\partial x}[(1-\alpha) u A]=0
$$




$$
\frac{\partial u}{\partial t}+u \frac{\partial u}{\partial x}=-\frac{1}{2(1-\alpha)} \frac{\partial C_{P}}{\partial x}
$$

where $\alpha(x, t)$, the bubble void fraction, is related to the bubble radius, $R(x, t)$, by $\alpha(x, t)=\frac{4}{3} \pi \eta R^{3}(x, t) /[1+$ $\left.\frac{4}{3} \pi \eta R^{3}(x, t)\right]$. The liquid has been assumed to be incompressible and the relative motion between the phases has been ignored. Friction between the fluid and the duct wall is also neglected. It is assumed that the upstream bubble population per unit volume of liquid is piecewise uniform and that there is no coalescence or break-up of bubbles in the flow. Since relative motion and the mass of liquid vaporized or condensed are neglected, it follows that $\eta$ remains both constant and piecewise uniform in the flow. The non-dimensional mixture density has been approximated by $\rho \approx(1-\alpha)$ in (1) and (2) since the liquid density is very much larger than the vapor density. The interactions of the bubbles with the flow are modeled by the Rayleigh-Plesset equation (Knapp et. al. 1970; Plesset and Prosperetti 1977) which connects the local mixture pressure coefficient, $C_{P}$, to the bubble radius, $R$ :

$$
\begin{aligned}
R \frac{D^{2} R}{D t^{2}} & +\frac{3}{2}\left(\frac{D R}{D t}\right)^{2}+\frac{\sigma}{2}\left(1-R^{-3 k}\right)+\frac{4}{R e} \frac{1}{R} \frac{D R}{D t} \\
& +\frac{2}{W e}\left(R^{-1}-R^{-3 k}\right)+\frac{1}{2} C_{P}=0
\end{aligned}
$$

where $D / D t=\partial / \partial t+u \partial / \partial x$ is the Lagrangian derivative, $\sigma$ is the cavitation number, $W e$ is the Weber number, and $R e$ is the Reynolds number. The partial pressure of noncondensable gas (it is assumed the mass of gas inside each bubble is constant) does not appear explicitly in (3) because the upstream equilibrium condition has been employed to eliminate this quantity. It has also been assumed that the non-condensable gas inside the bubbles behaves polytropically with an index $k$. If $k=1$, a constant bubble temperature is implied and $k=\gamma$, the ratio of specific heats of the gas, would model adiabatic behavior.

Equations (1), (2) and (3) represent a simple model of one-dimensional flowing bubbly mixture with nonlinear bubble dynamics. Previous investigations have examined the dispersion and stability properties of this model in the linear regimes (see, for example, Biesheuvel and Wijngaarden 1984; Morioka and Matsui 1980; Morioka and Toma 1984; Toma and Morioka 1986; Toma et. al. 1988). These results helped to identify the propagation modes and the dispersion characteristics of the acoustic waves in a flowing bubbly liquid. However, if the flow is accelerating, simple linearization of the equations of motion is impossible since the mean flow quantities are changing rapidly with both space and time. Analyses of the dynamics of this model then become significantly more complicated and new phenomena may be manifest due to the coupling of flow acceleration and bubble dynamics.

\section{STEADY STATE SOLUTIONS}

Only steady flows are considered in the present work. It is assumed that (1), (2) and (3) have steady state solutions for a constant mass flow rate with upstream conditions denoted by $p_{s}^{*}, u_{s}^{*}$, and $\rho_{s}^{*} \approx \rho_{L}^{*}\left(1-\alpha_{s}\right)=\rho_{L}^{*} /\left(1+\frac{4}{3} \pi \eta R_{s}^{*^{3}}\right)$ where $R_{s}^{*}$ and $\alpha_{s}$ are the upstream nuclei size and void fraction, respectively. After dropping all the partial time derivative terms, the governing equations become a system of ordinary differential equations with one independent variable, $x$ :

$$
\begin{gathered}
(1-\alpha) u A=\left(1-\alpha_{s}\right)=\text { constant } \\
u \frac{d u}{d x}=-\frac{1}{2(1-\alpha)} \frac{d C_{P}}{d x} \\
R\left(u^{2} \frac{d^{2} R}{d x^{2}}+u \frac{d u}{d x} \frac{d R}{d x}\right)+\frac{3 u^{2}}{2}\left(\frac{d R}{d x}\right)^{2}+\frac{4}{R e} \frac{u}{R} \frac{d R}{d x} \\
+\frac{2}{W e}\left(\frac{1}{R}-\frac{1}{R^{3 k}}\right)+\frac{\sigma}{2}\left(1-\frac{1}{R^{3 k}}\right)+\frac{1}{2} C_{P}=0
\end{gathered}
$$

The initial or upstream conditions are given by:

$$
R(x=0)=1, \quad u(x=0)=1, \quad C_{P}(x=0)=0
$$

We choose to examine a simple nozzle, $A(x)$, such that

$$
A(x)= \begin{cases}\sqrt{1+\frac{1}{2} C_{P M I N}\left[1-\cos \left(\frac{2 \pi x}{L}\right)\right]}, & 0 \leq x \leq L \\ 1 & ; x<0 \text { and } \\ & x>L\end{cases}
$$

This profile will produce a simple sinusoidal pressure distribution in the case of incompressible flow with the minimum pressure coefficient, $C_{P M I N}$, located at the nozzle throat. The value of $-C_{P M I N}$ relative to the cavitation number, $\sigma$, represents the intensity of tension in the flow. If $-C_{P M I N}$ is greater than the cavitation number, $\sigma$, the minimum mixture pressure experienced by the individual bubbles will be lower than vapor pressure and the bubbles will cavitate.

\section{RESULTS AND DISCUSSION}

A fourth order Runge-Kutta scheme was used to integrate equations (5) and (6). The following flow conditions were chosen to illustrate the computational results. A bubbly liquid, composed of air bubbles $(k=1.4)$ in water at $20^{\circ} \mathrm{C}\left(\rho_{L}^{*}=1000 \mathrm{~kg} / \mathrm{m}^{3}, S^{*}=0.073 \mathrm{~N} / \mathrm{m}\right)$, is flowing through a nozzle with profile given by equation (8); the non-dimensional length of the nozzle is $L=500$. The minimum pressure coefficient, $C_{P M I N}$, for the pure liquid flow is chosen as -1 . The upstream cavitation number, $\sigma$, is set at 0.8 , smaller than $-C_{P M I N}$ so that cavitation will occur. 


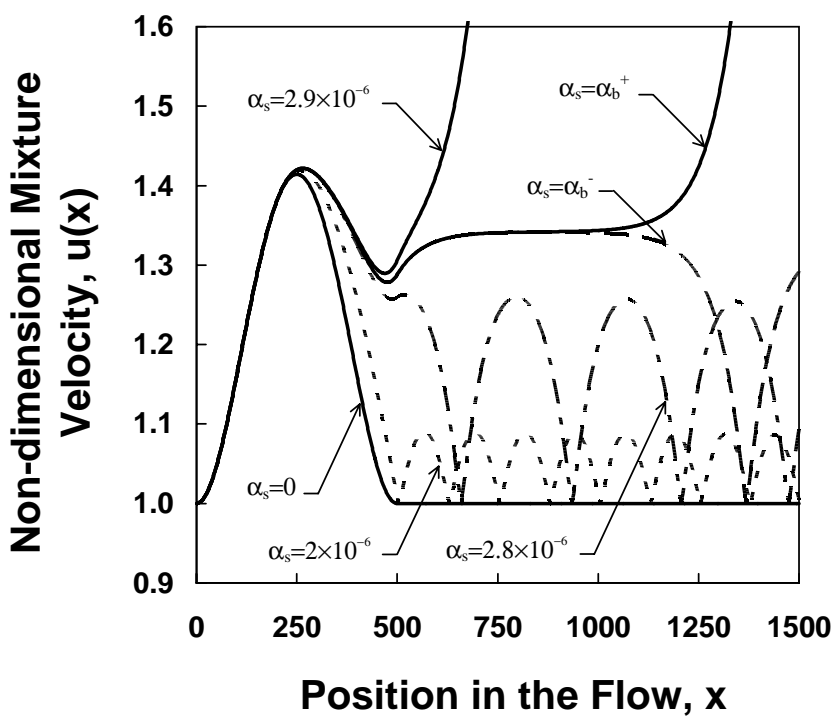

Figure 2: The non-dimensional mixture velocity distribution as a function of the non-dimensional position in the flow for five different upstream void fractions. Labels of $\alpha_{s}=\alpha_{b}^{-}$and $\alpha_{s}=\alpha_{b}^{+}$correspond to $\alpha_{s}$ just below and above the critical value $\alpha_{b} \approx 2.862 \times 10^{-6}$. The dimensionless length of the nozzle, $L$, is 500 with the throat located at 250. Other parameters are $\sigma=0.8, C_{P M I N}=-1.0$, $R e=1000$, and $W e=137$.

The Reynolds number, $R e$, is taken as 1000 ; this essentially determines the damping of the bubble oscillations. In the present example only the contribution of liquid viscosity to the damping is considered. Other damping mechanisms could be included by using an "effective viscosity" as described by Chapman and Plesset (1971). Five different upstream void fractions, $\alpha_{s}$, of the order of $10^{-6}$ are used in the computation and the results are shown in figures 2 to 5 .

Figure 2 illustrates the mixture velocity. The case of $\alpha_{s}=0$ corresponds to the incompressible pure liquid flow. It is notable that even for an upstream void fraction as small as $2 \times 10^{-6}$, the characteristics of the flow are radically changed from the case without bubbles. Radial pulsation of bubbles results in the downstream fluctuations of the flow. The amplitude of the velocity fluctuation is $10 \%$ of that of the incompressible flow in this case. As $\alpha_{s}$ increases further, the amplitude as well as the wavelength of the fluctuations increase. However, the velocity does eventually return to the upstream value. In other words, the flow is still "quasistatically stable." However, as $\alpha_{s}$ increases to a critical value, $\alpha_{b}$ (about $2.862 \times 10^{-6}$ in the present calculation), a bifurcation occurs. The velocity increases dramatically and the flow becomes "quasi-statically unstable." The physical

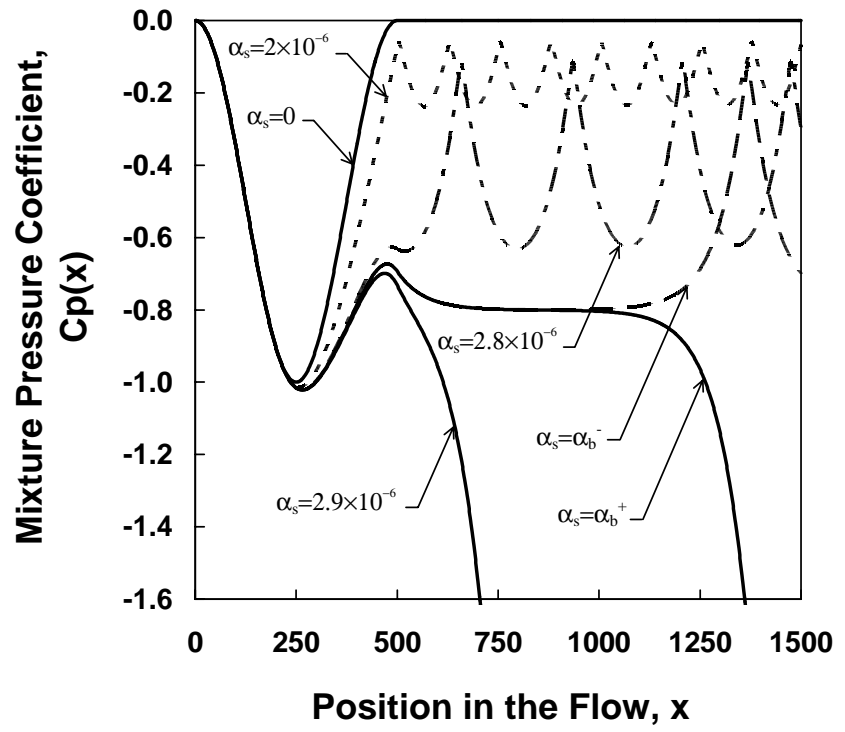

Figure 3: The mixture pressure coefficient corresponding to figure 2 .

picture of this instability is quite simple: Growth of the cavitation bubbles increases the mixture velocity according to the mass conservation of the flow. The increase of the velocity then causes the mixture pressure to decrease due to the Bernoulli effect. The decrease of the pressure is fed back to the Rayleigh-Plesset dynamics and results in more bubble growth.

The corresponding variations in the mixture pressure coefficient are shown in figure 3 . In addition to the two different flow regimes, another important feature in the quasistatically stable flow is the typical frequency associated with the downstream periodicity. This "ringing" will result in acoustic radiation at frequencies corresponding to this wavelength. How this ring frequency relates to the upstream flow condition remains to be studied. Furthermore, it should be noticed that there is a pressure loss downstream; the mixture pressure does not return to the upstream value except in the case of the pure liquid flow. The only damping mechanism in the present model is due to the liquid viscosity at the interface of the phases. Since the viscosity of wall and slip motion between bubbles and liquid are all neglected, the pressure loss is caused by the radial motion of bubbles and represents the "cavitation loss."

Figure 4 illustrates the void fraction distribution in the flow. When the flow becomes quasi-statically unstable, the bubble void fraction, $\alpha(x)$, quickly approaches unity. This means that the flow is flashing to vapor. Moreover we should emphasize that when $\alpha$ becomes large, our model equations, which are limited to flows with small void fraction (for the limitation of void fraction in the present model, 


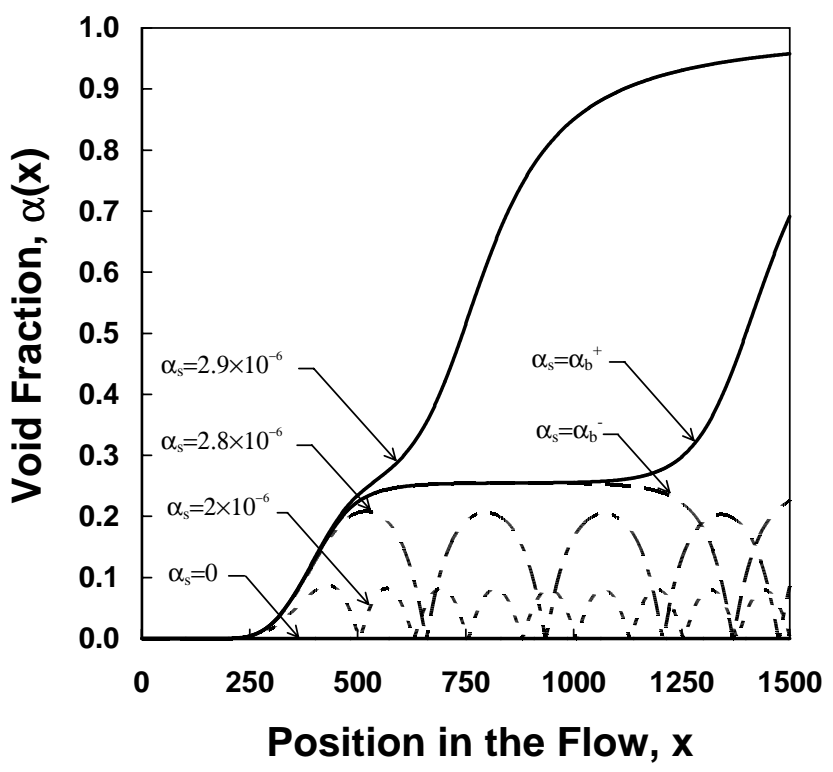

Figure 4: The void fraction distribution corresponding to figure 2 .

see d'Agostino and Brennen 1989), lose their validity.

Figure 5 indicates the non-dimensional bubble radius distribution in the flow. Due to time lag during the bubble growth phase, bubbles reach the maximum size after passing the nozzle throat. With increase in the upstream void fraction, the maximum size of the bubbles increases and is shifted further downstream. The bubbles grow without bound after reaching the critical radius, $R_{c}$, at which flashing begins. Note that $R_{c}$ is dependent on the cavitation number and the upstream void fraction. An analytical expression for $R_{c}$ can be found as follows. From figure 5 we note that $d R / d x$ and $d^{2} R / d x^{2}$ both vanish at $R=R_{c}$. Substitution of these conditions into (6) gives

$$
\frac{2}{W e}\left(R_{c}^{-1}-R_{c}^{-3 k}\right)+\frac{\sigma}{2}\left(1-R_{c}^{-3 k}\right)+\frac{1}{2} C_{P c}=0
$$

Here $C_{P_{c}}$ can be found by integrating (4) and (5) by putting $A=1$ (assuming that the flow exits the nozzle into a length of constant area duct downstream of the nozzle):

$$
C_{P c}=-\frac{24 \pi \eta R_{c}^{3}}{(3+4 \pi \eta)^{2}}\left(1-\frac{1}{R_{c}^{3}}\right)
$$

Since $R_{c} \gg 1$, all the higher order terms $\left(1 / R_{c}^{3 k}\right.$ in (9) and $1 / R_{c}^{3}$ in (10)) can be neglected. After combining these two equations, one can write

$$
R_{c}^{4}-\frac{\sigma}{2 \alpha_{b}\left(1-\alpha_{b}\right)} R_{c}-\frac{2}{\alpha_{b}\left(1-\alpha_{b}\right) W e}=0
$$

in which $\frac{4}{3} \pi \eta=\alpha_{b} /\left(1-\alpha_{b}\right)$ has been used. The third term in (11) can be neglected because, in addition to $R_{c} \gg 1$,

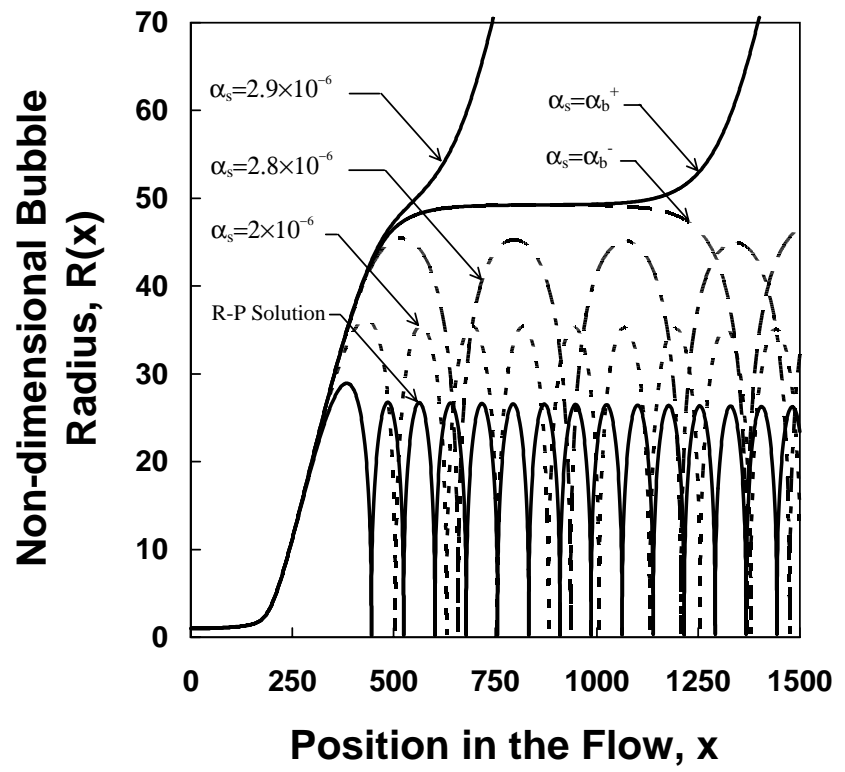

Figure 5: The non-dimensional bubble radius distribution corresponding to figure 2. R-P solution represents the solution from the Rayleigh-Plesset equation.

practical values for $2 / W e$ are about an order of magnitude less than the values of $\sigma / 2$ in the second term. Thus, finally we have:

$$
R_{c}=\left[\frac{\sigma}{2 \alpha_{b}\left(1-\alpha_{b}\right)}\right]^{1 / 3} \approx\left[\frac{\sigma}{2 \alpha_{b}}\right]^{1 / 3}
$$

If $R>R_{c}$, the flow becomes quasi-statically unstable and flashes. In the cases presented here $\left(\sigma / 2 \alpha_{b}\right)^{1 / 3} \approx 51$. Examination of figure 5 shows that this value is accurate. With known $R_{c}$, the expressions for the critical pressure coefficient can be obtained from (10):

$$
C_{P c}=2 \alpha_{b}\left(1-\alpha_{b}\right)-\sigma \approx-\sigma
$$

\section{CONCLUDING REMARKS}

Steady cavitating bubbly flows through a convergingdiverging nozzle have been examined in the present paper. It was found that the nonlinear bubble dynamics coupled with the equations of motion of the mixture strongly affect the structure of the flow even for very small bubble populations. Two different flow regimes, distinguished by the parameter $R_{c}=\left(\sigma / 2 \alpha_{b}\right)^{1 / 3}$, (where $\sigma$ is the cavitation number of the flow and $\alpha_{b}$ is the upstream void fraction at which the bifurcation occurs) are revealed in the steady state solutions. The flow becomes quasi-statically unstable and flashes to vapor if the radius of the cavitating bubbles 
is greater than $R_{c}$. In this circumstance, the growth of bubbles increases the mixture velocity due to mass conservation of the flow. The velocity increase then causes the mixture pressure to decrease according to the momentum equation. The decrease of the pressure is fed back to the RayleighPlesset equation and results in further bubble growth. In this case the velocity and void fraction of the mixture increase and the pressure coefficient of the flow decreases significantly below the upstream values and the flow flashes to vapor. On the other hand, if the bubbles do not grow beyond $R_{c}$, the flow is quasi-statically stable and is characterized by large amplitude spatial fluctuations downstream of the throat.

Finally we should note that some unsteady effects may have important consequences on the flow instability, for example, the ratio of the duration of the tension applied to the flow $\left(L^{*} / u_{s}^{*}\right)$ to the natural period of bubble oscillation $\left(\sqrt{\rho_{L}^{*} R_{s}^{*} / 3 k p_{s}^{*}}\right)$. In Wang (1996), it was shown that the nonlinear bubble dynamics cause substantial computational difficulties in solving the unsteady equations (1), (2) and (3). This generic problem must be solved before the unsteady characteristics can be explored.

\section{ACKNOWLEDGMENTS}

The authors are very grateful for the support for this research provided by the Office of Naval Research under Contract N00014-91-J-1295.

\section{References}

[1] Biesheuvel, A. and Wijngaarden, L. van (1984). Two phase flow equations for a dilute dispersion of gas bubbles in liquid. J. Fluid Mech., 148, 301-318.

[2] Brennen, C.E. (1995). Cavitation and bubble dynamics. Oxford University Press.

[3] Chapman, R.B. and Plesset, M.S. (1971) Thermal effects in the free oscillation of gas bubbles. ASME J. Basic Eng., 93, 373-376.

[4] d'Agostino, L. and Brennen, C. E. (1983). On the acoustical dynamics of bubble clouds. In ASME Cavitation and Multiphase Flow Forum, 72-75.

[5] d'Agostino, L. and Brennen, C. E. (1989). Linearized dynamics of spherical bubble clouds. J. Fluid Mech., 199, 155-176.

[6] Ishii, R., Umeda, Y., Murata, S., and Shishido, N. (1993). Bubbly flows through a converging-diverging nozzle. Phys. Fluids A, 5 (7), 1630-1643.
[7] Kameda, M. and Matsumoto, Y. (1995). Structure of shock waves in a liquid containing gas bubbles. In $I U$ TAM Symposium on Waves in Liquid/Gas and Liquid/Vapour Two-Phase Systems, pp. 117-126.

[8] Knapp, R.T., Daily, J.W., and Hammitt, F.G. (1970). Cavitation. Oxford University Press.

[9] Morioka, S. and Matsui, G. (1980). Mechanism and effect of dispersion and dissipation in nozzle flows of bubbly liquid. In Proc. 1st Asian Cong. Fluid Mech., A 54 .

[10] Morioka, S. and Toma, T. (1984). Stability of twophase liquid metal MHD channel flows. Prog. Astronaut. Aeronaut., 100, 317.

[11] Noordzij, L. \& Wijngaarden, L. van (1974). Relaxation effects, caused by relative motion, on shock waves in gas-bubble/liquid mixtures. J. Fluid Mech., 66, 115143.

[12] Plesset, M. S. and Prosperetti, A. (1977). Bubble dynamics and cavitation. Ann. Rev. Fluid Mech., 9, 145185.

[13] Tangren, R.F., Dodge, C.H., and Seifert, H.S. (1949). Compressibility effects in two-phase flow. J. Appl. Phys., 20, No. 7, 637-645.

[14] Toma, T. and Morioka, S. (1986). Acoustic waves forced in flowing bubbly liquid. J. Phys. Soc. Japan, 55, No. 2, 512-520.

[15] Toma, T., Yoshino, K., and Morioka, S. (1988). Fluctuation characteristics of bubbly liquid flow in converging-diverging nozzle. Fluid Dyn. Res., 2, 217228.

[16] Wang, Y.-C. (1996). Shock waves in bubbly cavitating flows. PhD thesis, California Institute of Technology, Pasadena, California.

[17] Wijngaarden, L. van (1968). On the equations of motion for mixtures of liquid and gas bubbles. J. Fluid Mech., 33, 465-474.

[18] Wijngaarden, L. van (1972). One-dimensional flow of liquids containing small gas bubbles. Ann. Rev. Fluid Mech., 4, 369-396. 